https://doi.org/10.15407/ujpe64.5.371

G.G. RODE

Institute of Physics, Nat. Acad. of Sci. of Ukraine

(46, Nauky Ave., Kyiv 03028, Ukraine; e-mail: ifanrode@gmail.com)

\title{
PROPAGATION OF THE MEASUREMENT \\ ERROR AND THE MEASURED MEAN OF A PHYSICAL QUANTITY FOR ELEMENTARY FUNCTIONS $a^{x}$ AND $\log _{a} x$
}

\begin{abstract}
Rules have been obtained for the propagation of the error and the mean value for a measured physical quantity onto another one with a functional relation of the type $a^{x}$ or $\log _{a} x$ between them. In essence, these rules are inherently based on the Gaussian weight scheme. Therefore, they should be valid in the framework of a real Gaussian weight scheme applied to discrete data of a real physical experiment (a sample). An analytical form that was used to present the rules concerned ("analytical propagation rules") and their character allow the processing and the analysis of experimental data to be simplified and accelerated.

Keywords: propagation of an error, propagation of an uncertainty.
\end{abstract}

\section{Introduction}

This work is devoted to an actual topic of estimating the errors for indirectly measured physical quantities. It makes a contribution to the general problem of error propagation. In more details, the problem of error propagation was described in works $[1,2]$. It was also discussed in work [3].

There are two approaches to solve this problem. All modern theoretical and practical applications, methods, and developments concerning the propagation of errors are based exclusively on the expansion in a Taylor series ("differentiation") [4-13]. The best description of the problem of "analytical" error propagation is given in work [1]. In this paper, some efforts in this direction were made. Namely, two widely used elementary functions, $a^{x}$ and $\log _{a} x$ (or, equivalently, $e^{x}$ and $\left.\ln x\right)$ were considered. This work is a logical continuation of works $[2,3]$.

(C) G.G. RODE, 2019

ISSN 2071-0194. Ukr. J. Phys. 2019. Vol. 64, No. 5

\section{New Rules for the Calculation} of Means and the Propagation of Errors in the Case of Elementary Functions $a^{x}$ and $\log _{a} x$

To obtain analytical rules for two examined functions $\left(a^{x}\right.$ and $\left.\log _{a} x\right)$, the mean $x_{a v}$ and the "error" $k \sqrt{(\Delta x)_{a v}^{2}}$ were related (formalized) to the basic notions of mathematical statistics:

$x_{a v} \approx E_{x}, \quad k^{2}(\Delta x)_{a v}^{2} \approx D_{x}$,

where $E_{x}$ and $D_{x}$ are, respectively, the mathematical expectation and the variance of the measured quantity $x$. At this formalization, it is assumed that, when measuring the physical quantity $x$, its specific values $x_{i}$ are obtained in accordance with a certain function $f(x)$ describing the probability for any $x$-value to appear. Surely, this function has to depend on the measurement conditions: it implicitly depends on the measuring device, the chosen method, and so forth). As a rule, the function $f(x)$ is 
normalized,

$\int_{-\infty}^{\infty} f(x) d x=1$

In this case, it is called the function of the probability density for a physical quantity $x$ with the continuous distribution. Accordingly, the true value $\mu$ of the physical quantity $x$ - or its mathematical expectation $E_{x}$ - can be calculated using the known function $f(x)$ by the formula

$\mu=E_{x}=\int_{-\infty}^{\infty} x f(x) d x$.

Equation (2) is a definition of the mathematical expectation $E_{x}$ [1]. The function $f(x)$ also determines the variance $D_{x}$ of the physical quantity $x$ [1] (the spread of its measured values owing to $f(x))$ :

$D_{x}=\int_{-\infty}^{\infty}\left(x-E_{x}\right)^{2} f(x) d x=\int_{-\infty}^{\infty}(x-\mu)^{2} f(x) d x$,

where $\mu=E_{x}$. The so-called normal (Gaussian) probability distribution [1]:

$f(x)=\frac{p}{\sqrt{\pi}} \exp \left[-p^{2}(x-\mu)^{2}\right]$,

where $p^{2}=\frac{1}{2 D_{x}}$ and $\mu=E_{x}$, is considered to be the most important one among other distributions.

In the case of a relationship by means of the function $y=h(x)$, the mathematical expectation $E_{h}$ and the variance $D_{h}$ for the function $h(x)$ equal [1], respectively,

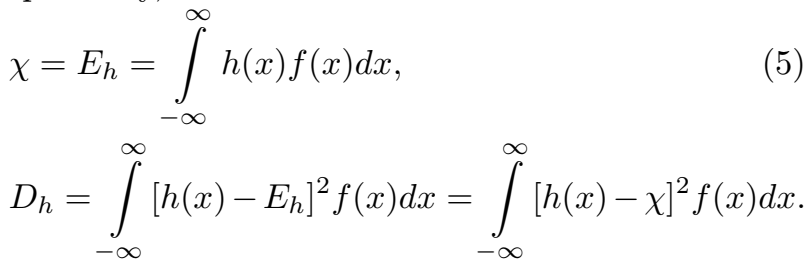

Expression (6) can be rewritten in a more convenient form,

$$
\begin{aligned}
& D_{h}=\int_{-\infty}^{\infty}\left[h^{2}(x)-2 h(x) E_{h}+E_{h}^{2}\right] f(x) d x= \\
& =\int_{-\infty}^{\infty} h^{2}(x) f(x) d x-E_{h}^{2} .
\end{aligned}
$$

In Eqs. (4)-(7), the quantities $\mu=E_{x}$ and $D_{x}$ enter to the function $f(x)$ as parameters. Therefore, strictly speaking, $f(x)$ can be written as $f\left(x, E_{x}, D_{x}\right)$. As a result,

$E_{h}=\int_{-\infty}^{\infty} h(x) f\left(x, E_{x}, D_{x}\right) d x$

$D_{h}+E_{h}^{2}=\int_{-\infty}^{\infty} h^{2}(x) f\left(x, E_{x}, D_{x}\right) d x$

It is easy to see that Eqs. (8) and (9) are integral equations. By solving them, we would obtain a sought analytic relation between $E_{h}$ and $D_{h}$ (analogs of the means for the function $h(x)$ ), on the one hand, and $E_{x}$ and $D_{x}$ (analogs of the measured averages), on the other hand. It turned out that it is possible to choose tabulated integrals [4] similar to integrals (8) and (9), and, in such a way, to solve the problem for the elementary function $a^{x}$ and, with its help, for the inverse function $\log _{a} x$ (see Appendix).

Here, a comment should be made. If the variable $x$ has a Gaussian distribution, the values of the nonlinear function $h(x)$ will be definitely not distributed according to the Gaussian law. Therefore, expressions (8) and (9) can be regarded as approximations to the expressions, in which the integration is carried out over the "correct" distribution:

$E_{h}=\int_{a}^{b} h Z\left(h, E_{h}, D_{h}\right) d h \quad D_{h}=$ $=\int_{a}^{b} h^{2} Z\left(h, E_{h}, D_{h}\right) d h-E_{h}^{2}$,

where $Z\left(h, E_{h}, D_{h}\right)$ is a "pure" non-Gaussian distribution for $h$. The integration limits $a$ and $b$ in these integrals depend on the function $h(x)$. For example, for $h=\cos x$, we have $a=-1$ and $b=1$.

However, the overwhelming majority of experimental works are based on the "classical" mixed summation scheme, Eqs. (8) and (9), which was expounded in work [1]. This is also true in the cases where Eqs. (8) and (9) are solved approximately by expanding $h(x)$ in the Taylor series [1]. Our expressions were also constructed in the framework of this ideology (see Appendix). Furthermore, all presented examples were also calculated, by using this scheme (see Section 3). In other words, both $x_{i}$ and $h\left(x_{i}\right)$ were summed up with the Gaussian probabilities.

ISSN 2071-0194. Ukr. J. Phys. 2019. Vol. 64, No. 5 
The means $E_{x}, D_{x}$, and $\Delta_{x}$ of the input set $\left\{x_{i}\right\}$ were calculated by the formulas

$\left.E_{x}=\sum x_{i} f_{i} ; \quad D_{h}=\sum\left(x_{i}-E_{x}\right)^{2} f_{i}\right) ; \Delta_{x}=\sqrt{D_{x}}$.

The means $E_{h}, D_{h}$, and $\Delta_{h}$ of the output set $\left\{h_{i}=h\left(x_{i}\right)\right\}$ were calculated by the formulas

$\left.E_{h}=\sum h\left(x_{i}\right) f_{i} ; \quad D_{h}=\sum\left(h\left(x_{i}\right)-E_{h}\right)^{2} f_{i}\right) ;$

$\Delta_{x}=\sqrt{D_{x}}$

where $f_{i}=\frac{w_{i}}{\sum w_{i}}$ are Gaussian multipliers, $f_{i}=\frac{w_{i}}{\sum w_{i}}$; $w_{i}=\frac{p}{\sqrt{\pi}} \times \exp \left[-p^{2}\left(x_{i}-\mu\right)^{2}\right], p^{2}=\frac{1}{2 D_{x}}$.

For the function $a^{x}$, the mentioned relations look like

$$
\begin{aligned}
& E_{h}=E_{a^{X}}=a^{E_{X}} a^{D_{X} \ln a / 2} \\
& D_{h}=D_{a^{X}}=a^{2 E_{X}} a^{D_{X} \ln a}\left(a^{D_{X} \ln a}-1\right),
\end{aligned}
$$

where $E_{X}$ and $D_{X}$ are the mean and the error, respectively, of the measured data, and $E_{a^{x}}$ and $D_{a^{x}}$ are the mean and the error, respectively, of the measurement results "propagated" with the use of the function $a^{x}$. For the function $\log _{a} x$, the analogous relations look like

$$
\begin{aligned}
& E_{h}=E_{\log }=\frac{1}{2} \log _{a}\left(\frac{E_{X}^{4}}{D_{X}+E_{X}^{2}}\right) ; \\
& D_{h}=D_{\log }=\frac{1}{\ln a} \log _{a}\left(\frac{D_{X}+E_{X}^{2}}{E_{X}^{2}}\right) ;
\end{aligned}
$$

where $E_{\log }$ and $D_{\log }$ are the mean and the error, respectively, of the measurement results "propagated" with the use of the function $\log _{a} x$. For the "pure" exponential function $h=\exp x$ and the "pure" logarithmic function $h=\ln x$, those formulas can be written in a simpler form:

- for $h=\exp x$,

$E_{h}=E_{\exp }=\exp E_{X} \exp \left(\frac{1}{2} D_{X}\right)$

$D_{h}=D_{\exp }=\exp 2 E_{X} \exp D_{X}\left(\exp D_{X}-1\right)$;

- for $h=\ln x$,

$$
\begin{aligned}
& E_{h}=E_{\ln }=\frac{1}{2} \ln \left(\frac{E_{x}^{4}}{D_{x}+E_{x}^{2}}\right) ; \\
& D_{h}=D_{\ln }=\ln \left(\frac{D_{x}+E_{x}^{2}}{E_{x}^{2}}\right) ;
\end{aligned}
$$

where $E_{\exp }$ and $D_{\exp }$ are the mean and the error, respectively, of the measurement results "propagated" with the use of the function $\exp x$, and $E_{\ln }$ and $D_{\ln }$ are their counterparts for the function $\ln x$.

Thus, we obtained (see Appendix) the sought rules for the error propagation and the calculation of "shifted means" of the type $E_{h}=E_{h}\left(E_{x}, D_{x}\right)$ and $D_{h}=D_{h}\left(E_{x}, D_{x}\right)$ for the functions $h(x)=a^{x}$ and $h(x)=\log _{a} x$ (in the particular case, $e^{x}$ and $\ln x$ ). Note that, in the limiting case $D_{x}=0$, we have

$$
\begin{array}{ll}
E_{h}=E_{a^{x}}=a^{E_{x}} ; & D_{h}=D_{a^{x}}=0 ; \\
E_{h}=E_{\log }=\log _{a} E_{x} ; & D_{h}=D_{\log }=0 ; \\
E_{h}=E_{\exp }=\exp E_{x} ; & D_{h}=D_{a^{x}}=0 ; \\
E_{h}=E_{\ln }=\ln E_{x} ; & D_{h}=D_{\ln }=0 .
\end{array}
$$

Therefore, the "standard" propagation rules can be applied:

$E_{a^{x}}=a^{E_{x}} ; \quad E_{\exp }=\exp E_{x} ; \quad E_{\log }=\log _{a} E_{x} ;$

$E_{\ln }=\ln E_{x}$.

\section{Application of the New Rules to Experimental Data}

A set of experimental data is a collection of separate random values $x_{i}$ obtained for a measured physical quantity $x$, i.e. the so-called "sample" $\left\{x_{i}\right\}$. The quantity $x$ may have a continuous distribution [1]; in other words, values that were randomly "selected by a measuring device" from a continuous set of values are dealt with.

Let us consider how the relations obtained above are satisfied just in the case of discrete samples. For this purpose, let us calculate the mean values, by using the standard procedure (which we take as a reference) for four samples: two sets of experimental data $\left\{x_{i}\right\}$, one set of calculated values for the $\exp x$ function, and one set of calculated values for the $\ln x$ function. Then we compare them with the results obtained according to relations (12) and (13).

\subsection{Two examples for the function $\exp x$}

As an example for the function $e^{x}$, let us take an arbitrary sample $\left\{x_{i}\right\}$ of 20 "measurements" on the logarithmic scale: $\left\{x_{i}\right\}=8,8.047,8.094,8.141,8.188,8.235,8.282$, $8.329,8.376,8.423,8,7.953,7.906,7.859,7.812$, $7.765,7.718,7.671,7.624$, and 7.577 .

The arithmetic means calculated for this sample with a constant probability $w_{i}=1 / 20$ give the following values: $E_{n}=8, D_{n}=0.06296$, and $\Delta_{n}=$ 


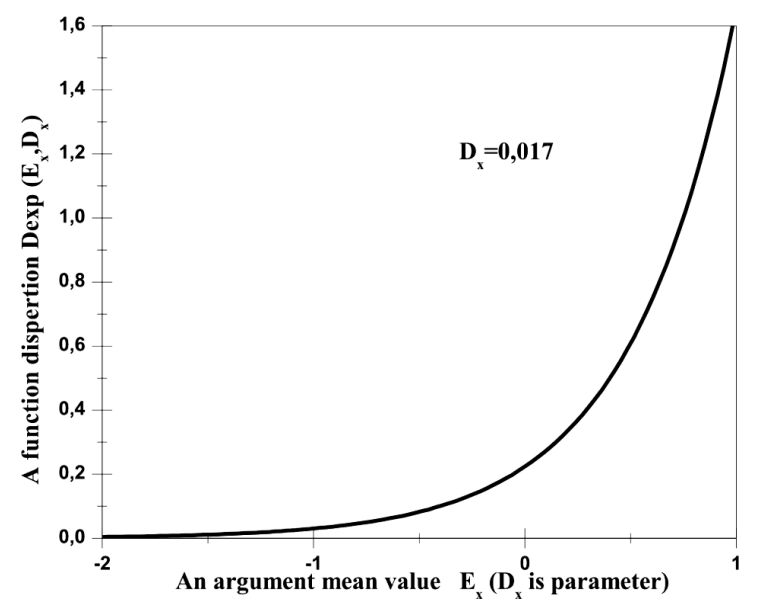

Fig. 1. Dependence of the variance $D_{\exp }$ of the function $\exp x$ on the mathematical expectation $E_{x}$ of its argument for the argument variance $D_{x}=0.017$

$=0.25092$. Using them as the first approximation, we calculate the Gaussian means (2 to 3 iterations) according to the Gauss weighted scheme

$$
\begin{aligned}
& \left.E_{x}=\left(\sum x_{i} w_{i}\right) / \sum w_{i}\right) ; \\
& \left.D_{x}=\left(\sum\left(x_{i}-E_{x}\right)^{2} w_{i}\right) / \sum w_{i}\right) ; \quad \Delta_{x}=\sqrt{D_{x}},
\end{aligned}
$$

where

$w_{i}=\frac{p}{\sqrt{\pi}} \exp \left[-p^{2}\left(x_{i}-\mu\right)^{2}\right], \quad p^{2}=\frac{1}{2 D_{x}}$.

As a result, we obtain $E_{x}=8, D_{x}=0.01726$, and $\Delta_{x}=0.13138$. In other words, we have $E_{x}=8 \pm 0.1$ for this sample.

In order to properly calculate the means for the function $e^{x}$, we should create a new statistical sample $\left\{\exp x_{i}\right\}$ and calculate its meaning values. The new sample looks like

$\left\{\exp x_{i}\right\}=2980.95799,3124.40767,3274.76045$, 3432.3485, 3597.52001, 3770.6399, 3952.09066, $4142.27321, \quad 4341.60772, \quad 4550.5346, \quad 2980.95799$, 2844.09445, 2713.51467, 2588.93015, 2470.06563, $2356.65849,2248.45816,2145.2256,2046.73271$, and 1952.76188 .

Hereafter, we intentionally left more significant digits than it is required (two for $D_{x}$ and one for $\Delta_{x}$ ) in order to give the readers an opportunity to trace the calculations. Using the values of $E_{x}, D_{x}$, and $\Delta_{x}$ together with formulas (14) and (15), we obtain the sought means for the function $e^{x}$ in the standard way: $E_{\exp }=3006.8068, D_{\exp }=157799.52$, and $\Delta_{\exp }=397.23987$. The "propagation of errors" according to the obtained relations (12) gives the following values: $E_{\exp }=3006.7946, D_{\exp }=157398.77$, and $\Delta_{\exp }=396.735$. Here, we have an interesting example with large dispersions for the function $e^{x}$.

Another example was created for a different domain of the argument of the function $e^{x}$ (Fig. 1):

$\left\{x_{i}\right\}=0,0.047,0.094,0.141,0.188,0.235,0.282$, $0.329,0.376,0.423,0,-0.047,-0.094,-0.141$, $-0.188,-0.235,-0.282,-0.329,-0.376,-0.423$.

The arithmetic means calculated for this sample with a constant probability $w_{i}=1 / 20$ give the following values: $E_{n}=0, D_{n}=0.06296$, and $\Delta_{n}=0.25091$. Using them as the first approximation, we calculate the Gaussian means (here, four iterations are required) according to the Gaussian weight scheme (14) and (15). As a result, we obtain $E_{x}=0, D_{x}=$ $=0.02194$, and $\Delta_{x}=0.14812$. In other words, we have $E_{x}=0 \pm 0.1$ for this sample.

In order to correctly calculate the means for the function $e^{x}$, we have to construct a new statistical sample $\left\{\exp x_{i}\right\}$ and calculate the required meanings for it. The new sample looks like

$\left\{\exp x_{i}\right\}=1,1.04812,1.09856,1.15142,1.20683$, $1.26491,1.32578,1.38958,1.45645,1.52653,1$, $0.95409,0.91028,0.86849,0.82861,0.79057,0.75427$, $0.71964,0.6866,0.65508$.

Using the values of $E_{x}, D_{x}$, and $\Delta_{x}$ together with formulas (14) and (15), we obtain the sought means for the function $e^{x}$ in the standard way: $E_{x}=1.01103$, $D_{x}=0.02269$, and $\Delta_{x}=0.15067$. The "propagation of errors" according to the obtained relations (12) gives the following values: $E_{\exp }=1.01103$, $D_{\text {exp }}=0.02267$, and $\Delta_{\text {exp }}=0.15058$.

Taking the both examples into account, we may assert that the values of errors (the variance and the deviation) evaluated by relation (12) are the same as for our reference examples. In other words, in the case of the function $e^{x}$, the propagation of errors with the use of relations (12) is proper.

\subsection{An example for the function $\ln x$}

Let us consider an example for the function $\ln x$ (Figs. 2 to 4). We use a sample of measurements of a quantity with the exponential dependence (say, the intensity measured, when determining the quantum yield of the activation energy):

$\left\{y_{i}\right\}=2000,2100,2200,2300,2400,2500,2600$, 2700, 2800, 2900, 2000, 1900, 1800, 1700, 1600, 1500, 1400, 1300, 1200, 1100.

ISSN 2071-0194. Ukr. J. Phys. 2019. Vol. 64, No. 5 


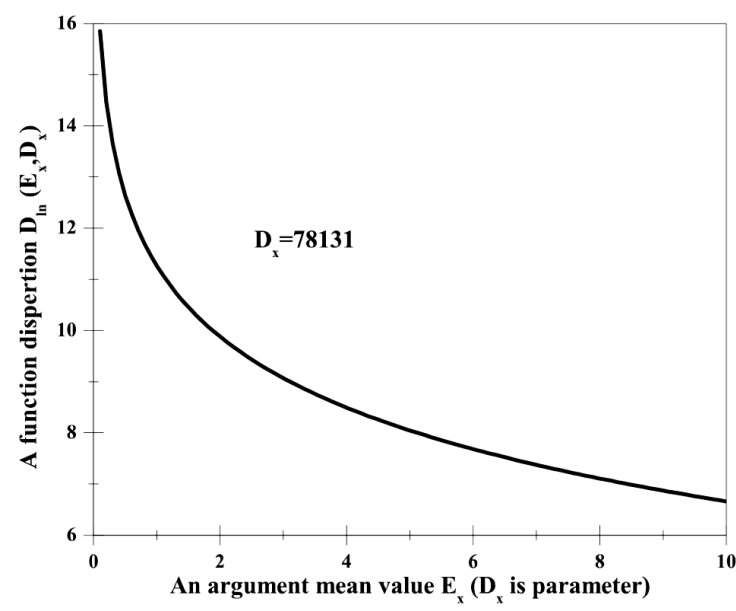

Fig. 2. Dependence of the variance $D_{\ln }$ of the function $\ln x$ on the mathematical expectation $E_{x}$ of its argument for the argument variance $D_{x}=78131.0$

Here, the variance of the argument is very large. Following the standard scheme for the calculation of means over the sample, we

(i) calculate the arithmetic means (with the probability $\left.w_{i}=1 / 20\right): E_{n}=2000, D_{n}=285000$, and $\Delta_{n}=533.8539$;

(ii) calculate the Gaussian means (according to the weight scheme (14): $E_{y}=2000, D_{y}=78130.595$, and $\Delta_{y}=279.5185$;

(iii) create a sample for the function $\ln y$ :

$\left\{\ln y_{i}\right\}=7.6009,7.64969,7.69621,7.74066,7.78322$, 7.82405, 7.86327, 7.90101, 7.9373, 7.97247, 7.6009, 7.54961, 7.49554, 7.43838, 7.37776, 7.31322, 7.24423, 7.17012, 7.09008, 7.00307;

(iv) statistically process this sample, by using the values obtained for $E_{y}, D_{y}$, and $\Delta_{y}$, and get $E_{\mathrm{ln}}=$ $=7.59081, D_{\ln }=0.02068$, and $\Delta_{\ln }=0.1438$.

Calculations by relations (13) give us the following values: $E_{\ln }=7.59123, D_{\ln }=0.019344$, and $\Delta_{\ln }=$ $=0.13908$. It is evident that the agreement is good in this case. In other words, the propagation of errors with the use of formulas (13) is proper for the function $\ln x$.

\section{Some General Features of the Obtained Relations}

The analytical form obtained for the rules of error propagation makes it easy to distinguish the specific features of corresponding relations and even to plot graphical dependences, which is very useful

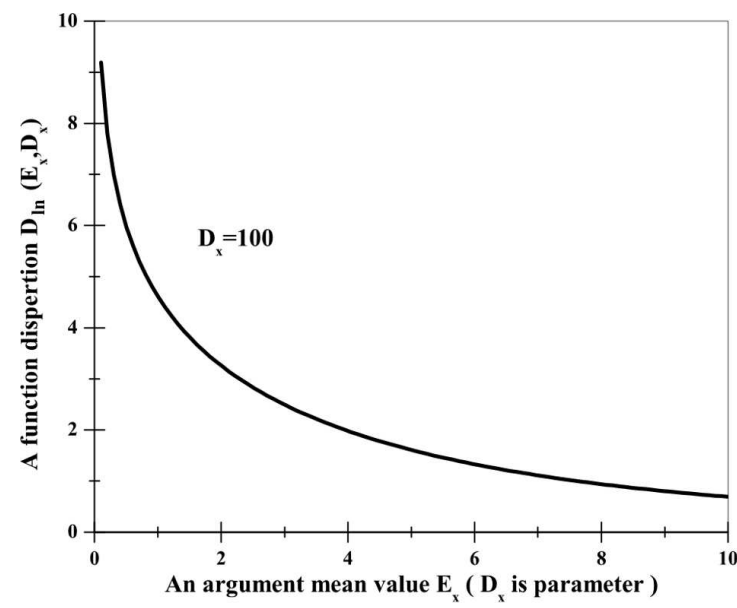

Fig. 3. Dependence of the variance $D_{\ln }$ of the function $\ln x$ on the mathematical expectation $E_{x}$ of its argument for the argument variance $D_{x}=100.0$

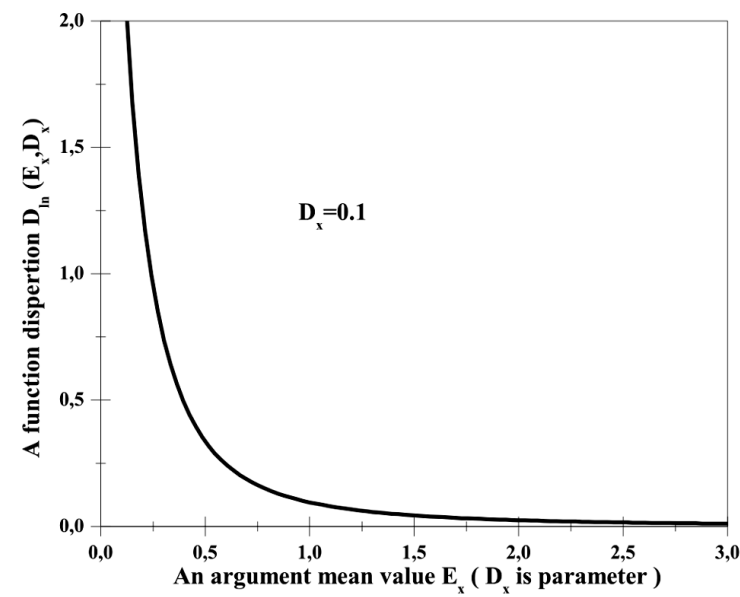

Fig. 4. Dependence of the variance $D_{\ln }$ of the function $\ln x$ on the mathematical expectation $E_{x}$ of its argument for the argument variance $D_{x}=0.1$

while planning a physical experiment and analyzing its results.

It should be emphasized that the quantities $E_{h}$, $D_{h}, E_{x}$, and $D_{x}$ are mutually related. Furthermore, the quantities $E_{h}$ and $D_{h}$ are functions of two arguments rather than one:

$E_{h}=E_{h}\left(E_{x}, D_{x}\right), \quad D_{h}=D_{h}\left(E_{x}, D_{x}\right)$.

It is sometimes difficult to get used to this fact, as well as to the fact that the errors of the functions $h(x)$, e.g., $\Delta_{\exp }$ or $\Delta_{\ln }$, depend on the measured mean value $x$. All those things are clearly illustrated in Figs. 1 to 
4, where the dependences of the function variances on the values of the measured "means" of the corresponding argument $E_{x}$ (the variance $D_{x}$ is regarded as a parameter) are depicted. The very opportunity to have a graphical representation for the obtained relations makes it possible to discuss the character of future measurements and plan them.

\section{Conclusions}

Similarly to what was done in work [6], relations (11) and (13) are considered to be semiempirical ones because of their construction on the basis of inverse functions. However, all inverse functions constructed in this way $[2,3]$, as well as relations (10)-(13) in this work, provide proper results for samples and can be widely used to shorten and to substantially simplify the calculation procedures for the functions $a^{x}$ and $\log _{a} x$. Since the variances $D_{h}$ and the errors $\sigma$ for both considered functions practically coincide with their real values, the propagation of errors can be performed in a chain of functions such as $\log _{a} b^{\log (\ldots)}, a^{\log _{b}() \cdots}$, or another combination of the indicated functions together with the functions $x^{2}$, $\sqrt{x}, \cos x$, and $\arccos x$ considered in works $[2,3]$.

Therefore, on the basis of the obtained analytical relations, it is possible to construct two simple general algorithms for the calculation of pairs of separate values $\left(E_{a^{x}}, D_{a^{x}}\right)$ and $\left(E_{\log }, D_{\log }\right)$ for the variable $x$ with the Gaussian distribution. They can be built-in as separate program modules (subroutines) into any program procedure. At the same time, this algorithm remains transparent for the understanding. This is impossible in principle for other error propagation methods, because they required the expansion in a series or the differentiation of the relevant superposition of functions as a whole entity. Moreover, every problem demands to construct a separate procedure.

The proposed method makes it possible to predict the error value of the function and to plot its profile in the intended measurement area of physical quantities.

Of interest is a possibility to obtain the shifted mean values for $E_{a^{x}}$ and $E_{\log }$. In the examples given above, this shift does not affect the values of those means and does not play any role, but it does exist, and its meaning can be used in some applications.

Since the analytical expressionfor the means $\left(E_{a^{x}}, D_{a^{x}}\right)$ and $\left(E_{\log }, D_{\log }\right)$ are connected with the Gaussian distribution, then the calculated values make it possible to compare them with the values of the same quantities, but calculated for other distributions. The best distribution will correspond to the minimum of $D_{a^{x}}$ or $D_{\log }$.

The materials of the cycle of works dealing with the estimation of errors for indirectly measured physical quantities were reported at the seminars of a number of departments at the Institute of Physics of the $\mathrm{Na}$ tional Academy of Sciences of Ukraine (Kyiv). The author is grateful to the teams and the heads of those departments (I.V. Blonskyi, M.S. Brodyn, A.G. Naumovets, A.M. Negryiko, Yu.O. Reznikov, S.M. Ryabchenko, P.M. Tomchuk, and L.P. Yatsenko) for their attention and an opportunity given to the author to look at the problem from their viewpoint. Without critical remarks expressed at those representations, the work, if any, would have been much worse. The author is especially thankful to S.M. Ryabchenko for his meticulous discussion of the results obtained, to G.V. Klimusheva for reading the paper and critical remarks, to O.I. Voitenko for his advice, as well as to everyone who was not indifferent and supported this work. The work was carried out in the framework of the budget theme 1.4.1. B/174 "Electrical, magnetic, and nonlinear optical properties of nano-dispersed particles of different origins in oriented liquid crystals".

\section{APPENDIX}

In this appendix, a mathematical proof is given that the relations obtained for two functions, $a^{x}$ and $\log _{a} x$, by reducing the integral equations (8) and (9) to tabulated integrals and transforming the obtained formulas to the convenient forms (10)-(13), are proper.

Mathematical expectation

$E_{h}$ for the function $h(x)=a^{x}$

By applying sequentially the substitutions $k=\ln a, h(x)=$ $=a^{x}=\exp (x \ln a)=\exp (k x), y=x-\mu$, and $x=y+\mu$ to Eq. (8) and taking the Gaussian distribution (4) for $h(x)$ into account, we can make the following chain of transformations:

$$
\begin{aligned}
& \chi=E_{h}=E_{a^{x}}=\frac{p}{\sqrt{\pi}} \int_{-\infty}^{\infty} a^{x} \exp \left[-p^{2}(x-\mu)^{2}\right] d x= \\
& =\frac{p}{\sqrt{\pi}} \int_{-\infty}^{\infty} \exp [k(y+\mu)] \exp \left[-p^{2} y^{2}\right] d y= \\
& =\frac{p}{\sqrt{\pi}} \exp [k \mu] \int_{-\infty}^{\infty} \exp \left[-p^{2} y^{2}+k y\right] d y=\frac{p}{\sqrt{\pi}} \exp [k \mu] J= \\
& =\frac{p}{\sqrt{\pi}} \exp [\ln a \mu] J=\frac{p}{\sqrt{\pi}} a^{\mu} J .
\end{aligned}
$$


The integral

$$
J=\int_{-\infty}^{\infty} \exp \left[-p^{2} y^{2}+k y\right] d y
$$

is similar to the tabulated integral [4, integral (3.923.2)]

$T_{2}=\int_{-\infty}^{\infty} \exp \left[-a_{1} y^{2}-2 b y-c\right] \cos \left[p^{2} y^{2}+q y+r\right] d y$.

The latter, at $c=p=q=r=0$, equals

$T_{2}=\int_{-\infty}^{\infty} \exp \left[-a_{1} y^{2}-2 b y\right] d y=\sqrt{\frac{\pi}{a_{1}}} \exp \frac{b^{2}}{a_{1}}$.

Hence, the integrals $J$ and $T_{2}$ are identical with an accuracy to the substitutions $a_{1}=p^{2},-2 b=k$, and $b=-\frac{k}{2}$. Therefore, taking into account that $k=\ln a$ and $1 / p^{2}=2 D_{x}$ [see Eq. (4)], we obtain

$J=\sqrt{\frac{\pi}{p^{2}}} \exp \frac{k^{2}}{4 p^{2}}=\frac{\sqrt{\pi}}{p} \exp \left[(\ln a)^{2} 2 D / 4\right]=$

$=\frac{\sqrt{\pi}}{p} a^{D \ln a / 2}$.

Substituting this expression into expression (16), we find a final relation of the integral $E_{h}=E_{a^{x}}$ to the integrals $E_{x}$ and $D_{x}$. Taking into account that $\mu=E_{x}$ [see Eq. (2)] and $p^{2}=$ $=1 /\left(2 D_{x}\right)[$ see Eq. (4)], this relation looks like

$E_{h}=E_{a} x=\frac{p}{\sqrt{\pi}} a^{\mu} \frac{\sqrt{\pi}}{p} a^{D_{X} \ln a / 2}=a^{\mu} a^{D_{X} \ln a / 2}=$

$=a^{E_{X}} a^{D_{X} \ln a / 2}$.

If $a=e$, then

$E_{h}=E_{\exp }=e^{E_{x}} e^{D_{x} / 2}$.

This is an "intuitively" expected result. As $D_{x} \approx 0$, there is a small shift associated with the multiplier $e^{D_{x} / 2} \approx 1$, and Eqs. (18) and (19) acquire a "natural" form

$E_{h}=E_{a^{x}} \approx a^{E_{x}}, \quad E_{h}=E_{\exp } \approx \exp E_{x}$.

Under certain conditions, this multiplier can be ignored, but Eqs. (18) and (19) are exact working formulas for $h(x)=a^{x}$ and $h(x)=\exp x$.

Variance $D_{h}$ for the function $h(x)=a^{x}$

From Eq. (9) with the use of Eq. (4), we obtain the following expression for the error propagation:

$D_{h}=D_{a^{x}}=\frac{p}{\sqrt{\pi}} \int_{-\infty}^{\infty} a^{2 x} \exp \left[-p^{2}(x-\mu)^{2}\right] d x-E_{h}^{2}=$

$=\frac{p}{\sqrt{\pi}} J_{0}-E_{h}^{2}$

Now, we transform

$J_{0}=\int_{-\infty}^{\infty} a^{2 x} \exp \left[-p^{2}(x-\mu)^{2}\right] d x$

ISSN 2071-0194. Ukr. J. Phys. 2019. Vol. 64, No. 5 into a tabulated integral, by using the sequential substitutions $y=x-\mu, x=y+\mu$, and $k=\ln a$ [see Eq. (8)]:

$J_{0}=\int_{-\infty}^{\infty} a^{2 x} \exp \left[-p^{2}(x-\mu)^{2}\right] d x=$

$=\int_{-\infty}^{\infty} a^{2 y+2 \mu} \exp \left[-p^{2} y^{2}\right] d y=$

$=a^{2 \mu} \int_{-\infty}^{\infty} a^{2 y} \exp \left[-p^{2} y^{2}\right] d y=$

$=a^{2 \mu} \int_{-\infty}^{\infty} \exp [\ln a 2 y] \exp \left[-p^{2} y^{2}\right] d y=$

$=a^{2 \mu} \int_{-\infty}^{\infty} \exp \left[-p^{2} y^{2}+2 k y\right]=a^{2 \mu} J_{01}$.

It is evident that

$J_{01}=\int_{-\infty}^{\infty} \exp \left[-p^{2} y^{2}+2 k y\right] d y$

is also integral (17) with $b=-k=-\ln a, a_{1}=p^{2}$, and $1 / p^{2}=$ $2 D_{x}$. Therefore,

$J_{01}=\frac{\sqrt{\pi}}{p} a^{2 D_{x} \ln a}$.

Using this result and Eq. (18) for $E_{h}$, from Eqs. (20) and (21), we obtain the variance $D_{h}$ for the function $h(x)=a^{x}$ in the form

$D_{h}=D_{a^{x}}=a^{2 E_{x}} a^{D_{x} \ln a}\left(a^{D_{x} \ln a}-1\right)$.

In the case $a=e$, we have a simplified form

$D_{h}=D_{\exp }=e^{2 E_{x}} e^{D_{x}}\left(e^{D_{x}}-1\right)$.

This formula is the error propagation rule for the function $h(x)=a^{x}$.

The mean $E_{h}$ and the variance

$D_{h}$ for the function $h(x)=\log _{a} x$

The straightforward calculation of the quantities $E_{\log _{a} x}$ and $D_{\log _{a} x}$ with the help of tabulated integrals is a rather problematic task. The sought relations can be obtained by considering the function $\log _{a} x$ as an inverse function to the function $a^{x}$ and by using Eqs. (18) and (22). Really, these equations give us an explicit relation between four integrals (numbers) $-E_{a^{x}}$, $D_{a} x, E_{x}$, and $D_{x}$ :

$E_{a^{x}}=E_{a^{x}}\left(E_{x}, D_{x}\right), \quad D_{a^{x}}=D_{a^{x}}\left(E_{x}, D_{x}\right)$.

Equations (18) and (22) can also be used to determine the functions

$E_{x}=E_{x}\left(E_{a^{x}}, D_{a^{x}}\right), \quad D_{x}=D_{x}\left(E_{a^{x}}, D_{a^{x}}\right)$, 
which are inverse to functions (24) and also have to properly describe the mathematical relations among $E_{a^{x}}, D_{a^{x}}, E_{x}$, and $D_{x}$. Furthermore, if $E_{a^{x}}$ and $D_{a^{x}}$ are obtained in a different way (e.g., they can be measured experimentally) and have the same numerical values as were calculated from formulas (24), then they will again satisfy Eqs. (8) and (9) that describe the relations among four integrals if and only if the quantities $E_{x}$ and $D_{x}$ have the same values that are given in Eq. (24).

In other words, if we have the relation $y=a^{x}$ and the inverse one $x=\log y$, then relations (25) inverse to Eqs. (18) and (22) will give us the proper values of integral expressions for the mathematical expectation $E_{x}$ and the variance $D_{x}$, determined by Eqs. (8) and (9), for the function of the random variable $y$ that is connected with the variable $x$ by the law $y=a^{x}$ (or $x=$ $\log _{a} y$ ). Hence, by solving Eqs. (18) and (22) with respect to $x$, we can obtain, by using a simple calculation procedure, the values of $E_{x}$ and $D_{x}$ from the values of $E_{y}$ and $D_{y}$, which are the means for the measured random variable $y$ that is related to $x$ by the formula $x=\log _{a} y$.

In order to solve Eqs. (18) and (22), let us rewrtite them in the form

$a^{D_{x} \ln a}=\frac{E_{y}^{2}}{a^{2 E_{x}}}$

and

$D_{y}=D_{a^{x}}=a^{2 E_{x}} a^{D_{x} \ln a}\left(a^{D_{x} \ln a}-1\right)$,

respectively. Bearing in mind that the integrals $E_{y}$ and $D_{y}$ are associated with the function $y=a^{x}$, whereas the integrals $E_{x}$ and $D_{x}$ with the function $y=\log _{a} x$, let us solve these equations with respect to the integrals $E_{x}$ and $D_{x}$, i.e. let us obtain the equations inverse to Eqs. (18) and (22), and to Eqs. (26) and (27). Substituting Eq. (26) into Eq. (27), we obtain

$D_{y}=E_{y}^{2}\left(\frac{E_{y}^{2}}{a^{2} E_{X}}-1\right)$,

whence

$\frac{D_{y}}{E_{y}^{2}}+1=\frac{D_{y}+E_{y}^{2}}{E_{y}^{2}}=\frac{E_{y}^{2}}{a^{2 E_{x}}}$,

so that

$a^{2 E_{x}}=\frac{E_{y}^{4}}{D_{y}+E_{y}^{2}}$

and, finally,

$E_{x}=\frac{1}{2} \log _{a}\left(\frac{E_{y}^{4}}{D_{y}+E_{y}^{2}}\right)$.

Substituting expression (28) into Eq. (26), after a series of elementary transformations, we obtain the final expression for $D_{x}$ :

$D_{x}=\frac{1}{\ln a} \log _{a}\left(\frac{D_{y}+E_{y}^{2}}{E_{y}^{2}}\right)$.

Let us rewrite the obtained relations (18), (23), (29), and (30) in a symbolic form, where $x$ is a measured physical quantity (argument), and $h$ is the corresponding function $\left(a^{x}\right.$, $\log _{a} x, e^{x}$, or $\left.\ln x\right)$ :

$E_{h}=E_{a}=a^{E_{X}} a^{D_{X}} \ln a / 2 ;$

$D_{h}=D_{a} X=a^{2 E_{X}} a^{D_{X} \ln a}\left(a^{D_{X} \ln a}-1\right)$;

$E_{h}=E_{\log }=\frac{1}{2} \log _{a}\left(\frac{E_{X}^{4}}{D_{X}+E_{X}^{2}}\right)$;

$D_{h}=D_{\log }=\frac{1}{\ln a} \log _{a}\left(\frac{D_{X}+E_{X}^{2}}{E_{X}^{2}}\right)$.

For the "pure" exponential function $\left(h(x)=e^{x}\right.$ or $\left.\ln x\right)$, these expressions look like

$E_{h}=E_{\exp }=\exp E_{X} \exp \left(\frac{D_{X}}{2}\right)$

$D_{h}=D_{\exp }=\exp 2 E_{X} \exp D_{X}\left(\exp D_{X}-1\right) ;$

$E_{h}=E_{\ln }=\frac{1}{2} \ln \left(\frac{E_{X}^{4}}{D_{X}+E_{X}^{2}}\right)$

$D_{h}=D_{\ln }=\ln \left(\frac{D_{X}+E_{X}^{2}}{E_{X}^{2}}\right)$.

With regard for the results of works [2,3], it is useful to reproduce the "analytic" rules of error propagation for two other pairs of functions:

for $\cos x$ and $\arccos x$,

$E_{h}=E_{\mathrm{cos}}=\exp \left(-\frac{D_{x}}{2}\right) \cos E_{x}$

$D_{h}=D_{\cos }=\frac{1}{2}\left[1-\exp \left(-D_{x}\right)\right]\left[1-\exp \left(-D_{x}\right) \cos 2 E_{x}\right] ;$

$E_{h}=E_{\arccos }=\arccos \frac{E_{x}}{ \pm \sqrt{E_{x}^{2}+\sqrt{\left(1-E_{x}^{2}\right)^{2}-2 D_{x}}}}$

$D_{h}=D_{\arccos }=\ln \left(\frac{1}{E_{x}^{2}+\sqrt{\left(1-E_{x}^{2}\right)^{2}-2 D_{x}}}\right)$.

and for $x^{2}$ and $\sqrt{x}$,

$E_{x^{2}}=E_{x}^{2}+D_{x}$,

$D_{x^{2}}=2 D_{x}^{2}+4 E_{x}^{2} D_{x}$,

$E_{\sqrt{x}}^{4}=E_{x}^{2}-\frac{1}{2} D_{x}$

$D_{\sqrt{x}}=E_{x}-\sqrt{E_{x}^{2}-\frac{1}{2} D_{x}}$.

In view of the formalization

$x_{a v} \approx E_{x}, \quad k^{2}(\Delta x)_{a v}^{2} \approx D_{x}$,

the obtained relations give us the sought "propagation rules" for the means and the errors of the functions $a^{x}, \log _{a} x, e^{x}$, $\ln x, x^{2}, \sqrt{x}, \cos x$, and $\arccos x$ :

$X_{a v} \rightarrow H_{a v}, \quad|\Delta X|_{a v} \rightarrow|\Delta H|_{a v}$.

1. D.J. Hudson, Statistics. Lectures on Elementary Statistics and Probability (CERN, 1964).

2. G.G. Rode. Propagation of measurement errors and measured means of a physical quantity for the elementary functions $\cos x$ and $\arccos x$. Ukr. J. Phys. 61, 345 (2016).

ISSN 2071-0194. Ukr. J. Phys. 2019. Vol. 64, No. 5 
3. G.G. Rode. Propagation of the measurement errors and measured means of physical quantities for the elementary functions $x^{2}$ and $\sqrt{x}$. Ukr. J. Phys. 62, 148 (2017).

4. I.S. Gradshtein, I.M. Ryzhik. Table of Integrals, Series, and Products (Academic Press, 1980).

5. Propagation of uncertainty [https://en.wikipedia.org/ wiki/Propagation of uncertainty].

6. H.H. Ku. Notes on the use of propagation of error formulas. J. Res. Nat. Bur. Stand. C 70, 263 (1966).

7. Ph.R. Bevington, D.K. Robinson. Data Reduction and Error Analysis for the Physical Sciences (McGraw-Hill, 2002).

8. J.R. Taylor. An Introduction to Error Analysis: The Study of Uncertainties in Physical Measurements (University Sci. Books, 1997).

9. B.N. Taylor, C.E. Kuyatt. Guidelines for Evaluating and Expressing the Uncertainty of NIST Measurement Results, NIST Technical Note 1297 (National Institute of Standards and Technology, 1994).

10. P.K. Sinervo. Denition and treatment of systematic uncertainties in high energy physics and astrophysics. In: Proceedings of the PHYSTAT2003 Conference, SLAC, Stanford, Ca, September 8-11 (2003), p. 122.

11. J. Denker. Nonlinear least squares [http://www.av8n.com/ physics/nonlinear-least-squares.htm]
12. E.W. Weisstein. Standard Deviation Entry at Math World [http://mathworld.wolfram.com/StandardDeviation.html.]

13. Evaluation of measurement data - An introduction to the "Guide to the expression of uncertainty in measurement" and related documents [http://www.bipm.org/utils/ common/documents/jcgm/JCGM 1042009 E.pdf].

Received 26.05.17. Translated from Ukrainian by O.I. Voitenko

Г.Г. Роде

ПЕРЕНОС ПОХИБОК

ТА СЕРЕДНІХ ВИМІРІВ ФІЗИЧНОЇ ВЕЛИЧИНИ

ДЛЯ ЕЛЕМЕНТАРНИХ ФУНКЦІЙ $a^{x}$ ТА $\log _{a} x$

$\mathrm{P}$ е $з$ ю м е

Отримані "правила переносу похибки та середнього" однієі вимірюваної фізичної величини на іншу, пов'язану з нею зв'язком $a^{x}$ або $\log _{a} x$. В ці правила по природі закладена вагова схема Гауса. Тому вони мають добре працювати в рамках реальної вагової схеми Гауса 3 дискретними даними реального фізичного дослідження (з “вибірками"). Аналітична форма, в якій представлені згадані правила ("аналітичні правила переносу"), а також їх характер дозволяють спростити і прискорити процедуру обробки й аналізу експериментальних даних. 